MATEC Web of Conferences 25, 02014

(2015)

DOI: $10.1051 /$ matecconf/ 20152502014

(C) Owned by the authors, published by EDP Sciences, 2015

\title{
The Effect of Explosion-suppression Materials on the Effective Volume and Cook-off Properties of Fuel Tank
}

\author{
Zhenli Yang \\ School of Chemical Engineering, Nanjing University of Science and Technology, Nanjing, Jiangsu, China \\ Youjie Zhou, Changbo Lu, Gaojun An, Chunhua Xiong \& Xudong Wang \\ Oil Research Institute of General Logistics Department, Beijing, China \\ Lifeng Xie \\ School of Chemical Engineering, Nanjing University of Science and Technology, Nanjing, Jiangsu, China
}

\begin{abstract}
In this paper, three kinds of explosion-suppression materials including aluminum alloy, polyurethane foam and spherical polymer are measured to get the data of displacement ratio and retention ratio, which can be used to calculate the reduction ratio of effective volume of the oil tank. The results show that the displacement ratio of the three kinds of materials are respectively $2.0 \%, 2.5 \%$ and $5.2 \%$, the retention ratio are respectively $1.1 \%, 3.2 \%$ and $0.4 \%$ and the reduction ratio of effective volume are respectively $3.1 \%, 5.7 \%$ and $5.6 \%$. Meanwhile, the cook-off properties of the oil tank filled with spherical high molecular explosion-suppression materials were tested, the methanol in the tank didn't explode, and materials in the tank didn't deform and be destroyed during the experiments. All of the results showed that the spherical high molecular explosion-suppression materials presented a good explosion-suppression performance.
\end{abstract}

Keywords: explosion-suppression materials; reduction ratio of effective volume; cook-off properties; explosion-suppression performance

\section{INTRODUCTION}

In the war, the fuel tanks of armored vehicles, tanks, warships are easily attacked by the shellfire, especially the secondary explosion of the fuel aerosol caused by the fire attack; it will lead to casualties and equipment damage which account for $50 \%$ of the total. The oil tank filled with the explosion-suppression materials can effectively protect the equipment against secondary explosion after the fire-fighting. So the adoption of explosion-suppression materials can enhance the survival rate of war fighters and lower the equipment damage rate significantly, improving the combat capability of army ${ }^{[1]}$. The explosion-suppression material is filled in the oil tank to separate the whole space of the tank to millions of small interspaces which can block-up the flame propagation, suppress the formation of explosion shock wave and prevent the instantaneous release of energy, leading to the explosion-suppression properties of the materials. These materials can be filled in the oil tanks of vehicles, aircrafts, ships and even pipeline of other facilities. Furthermore, the heat conduction effect of the material are also utilized to break up the explosive condition of flammable vapors preventing further combustion and explosion, and ensuring the security of storage, transportation and usage of the flammable and explosive oil chemical products. The normal explosion-suppression material consists of two categories, i.e. metal and nonmetal. It can also be divided into honeycomb type and ball type according to the shape of materials [1-4]. In daily life, the explosion-suppression material is applied to solve the problems of dangerous chemicals transportation and unexpected fuel burning and explosion. However, the utilization of explosion-suppression material filled in oil tanks or fuel tanks should meet a basic requirement, i.e. the original oil tank volume cannot be changed obviously. The US military standard MIL-B-87162A and MIL-PRF-87260B have given the requirements of the effective volume reduction of tank caused by the use of polyurethane explosion-suppression material and aluminum alloy explosion-suppression material in aviation fuel tank respectively ${ }^{[5]}$. In this paper, the replacement ratio and retention ratio of three kinds of explosion-suppression materials were measured, and then the reduction ratio of effective volume has been calculated of oil tank and fuel tank. Meanwhile, the cook-off performance of oil tanks which were filled with spherical high molecular explosion-suppression materials was tested to research its explosion-suppression properties.

\section{EXPERIMENT}

\subsection{Effective volume test of oil tank}

The replacement ratio of aluminum alloy explo- 


\section{MATEC Web of Conferences}

sion-suppression material, polyurethane foam explosion-suppression material and spherical high molecular explosion-suppression material were experimented according to MIL-T-5624. The samples were placed at the temperature of $20^{\circ} \mathrm{C}$ for 30 minutes. A standard cylinder which has a capacity of $2,000 \mathrm{~mL}$ was used in this experiment. The tested fuel was injected into the cylinder at $1800 \mathrm{~mL}$ scale line, and then the materials sample was slowly immersed in the fuel until completely infiltrated. For the different kinds of materials, the samples should be pretreated in order to be suitable to be positioned in the cylinder. The soaking time for the polyurethane foam explosion-suppression material is $24 \mathrm{~h}$ and that for the aluminum alloy explosion-suppression material and spherical high molecular explosion-suppression material is $10 \mathrm{~min}$. After that, the new height level of the fuel was recorded as the new volume of the cylinder after using the explosion-suppression materials. The replacement ratio of the explosion-suppression materials equals the difference of the new volume and the original volume of the cylinder.

The retention ratio of three kinds of materials is tested according to MTL-T-5624. The fuel samples used in this test should be filtered by a filter paper with a pore size of 0.8 micrometer. At first, the explosion-suppression material samples were placed at temperature of $20 \pm 1^{\circ} \mathrm{C}$ for 30 minutes, then the materials was weighted and put into a vessel with the size of $18 \times 18 \times 25 \mathrm{~cm}$. The material should be positioned on the vessel bottom and required a $1.3 \mathrm{~cm}$ distance to the vessel wall. For the different kinds of materials, the samples should be pretreated in order to be suitable to be positioned in the vessel. Then the fuel was poured into the square container, making sure that the liquid level of fuel which exceeded the top of material was more than $1.27 \mathrm{~cm}$ in height. The fuel was drained at the rate of $0.5 \pm 0.05 \mathrm{~L} / \mathrm{min}$ by adjusting the valve in the bottom of container. The materials were kept in the vessel for 2 minutes and then put out for weighting. The retention ratio was calculated using the following formula:

$$
C=\left(C_{2}-C_{1}\right) /\left(V_{3} \bullet \rho_{2}\right) \times 100
$$

Where:

$C$ is the retention ratio (\%);

$C_{1}$ is the mass of explosion-suppression material sample before immersion $(g)$;

$C_{2}$ is the mass of explosion-suppression material sample after immersion $(g)$;

$V_{3}$ is the volume of explosion-suppression material sample $\left(\mathrm{cm}^{3}\right)$;

$\rho_{2}$ is the density of contact medium $\left(\mathrm{g} / \mathrm{cm}^{3}\right)$.

\subsection{Cook-off properties of oil tank}

The cook-off test usually can be divided as slow cook-off test and fast cook-off test. The heating rate of slow cook-off test is generally less than $0.05^{\circ} \mathrm{C} / \mathrm{S}$ and the heating rate of fast cook-off test which is more than $1^{\circ} \mathrm{C} / \mathrm{S}$.

The fast cook-off test was originally designed to simulate the thermal response of ammunition under fire conditions and evaluate its thermal safety. Generally, different organic fuels such as gasoline, alcohol, wood or other heating materials were used to heat the measured sample directly during the test according to GB/T14372-2005 Recognition Component Test Method and Criteria of Transporting Explosives on Dangerous Goods. Because of the strict requirement for testing environments, the results were greatly influenced by natural condition, so the method was mainly used for the testing of whole missile or some energetic materials with large volume. It can also be applied in the thermal grill test of pint-sized energetic materials by using some improved burning test devices ${ }^{[6,7]}$.

In this paper, the cook-off test was conducted to research whether the fuel tank containing methanol would explode or not when filled with explosion-suppression material. The main equipment used in this testing was a steel tank (thickness of $6 \mathrm{~mm}$ ) with the volume of $100 \mathrm{~L}$. The size of steel burning pool was $1500 \times 450 \times 400 \mathrm{~mm}$. The test device is shown in Figure 1:
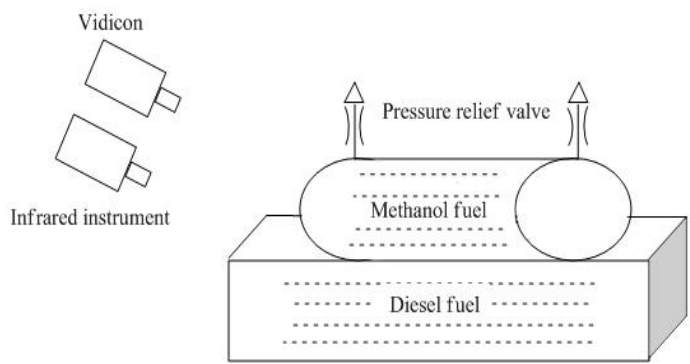

Figure 1. Site layout diagram of cook-off test

The spherical high molecular explosion-suppression material was loaded into the tank with the standard of $60 \mathrm{~kg} / \mathrm{m}^{3}$. After that the diesel fuel in the burning pool was ignited through the ignition system, the fuel tank would be observed by vidicons and infrared thermal imagers from a safe distance.

\section{ANALYSIS AND DISCUSSION}

\subsection{Effective volume of oil tank}

The replacement ratio and retention ratio of aluminum alloy explosion-suppression material, polyurethane foam explosion-suppression material and spherical high molecular explosion-suppression material are 
EMME 2015

Table 1. Replacement ratio and retention ratio of explosion-suppression materials

\begin{tabular}{|c|c|c|c|c|c|c|c|c|c|}
\hline \multirow{2}{*}{$\begin{array}{l}\text { Explosion-suppression } \\
\text { material }\end{array}$} & \multicolumn{3}{|c|}{$\begin{array}{l}\text { Replacement ratio } \\
(\%)\end{array}$} & \multirow[t]{2}{*}{ Average } & \multicolumn{3}{|c|}{$\begin{array}{l}\text { Retention ratio } \\
(\%)\end{array}$} & \multirow[t]{2}{*}{ Average } & \multirow{2}{*}{$\begin{array}{l}\text { Reduction ratio of effec- } \\
\text { tive volume }(\%)\end{array}$} \\
\hline & 1 & 2 & 3 & & 1 & 2 & 3 & & \\
\hline Aluminum alloy & 1.9 & 2.2 & 1.9 & 2.2 & 1.2 & 1.0 & 1.1 & 1.1 & 3.3 \\
\hline Polyurethane foam & 2.3 & 2.6 & 2.6 & 2.5 & 4.3 & 4.2 & 4.1 & 4.2 & 6.7 \\
\hline Spherical polymer & 5.4 & 5.0 & 5.2 & 5.2 & 0.4 & 0.3 & 0.5 & 0.4 & 5.6 \\
\hline
\end{tabular}

Table 2. Character of cook-off test

Table 2. Character of cook-off test
\begin{tabular}{|l|l|}
\hline Test of time & Phenomenon \\
\hline $4 \mathrm{~min}$ & Pressure relief valve spurted flame and liquid methanol seen in Figure 3a. \\
\hline $10 \mathrm{~min}$ & Pressure relief valve sharply spurted flame and spray of methanol seen in Figure $3 \mathrm{~b}$. \\
\hline $15 \mathrm{~min}$ & The flame and spray of methanol recede seen in Figure $3 \mathrm{c}$. \\
\hline $23 \mathrm{~min}$ & Pressure relief valve stopped spurting flame seen in Figure $3 \mathrm{~d}$. \\
\hline
\end{tabular}

shown in Table 1.

From Table 1, we can see that among the three kinds of explosion-suppression material, the replacement ratio of metal explosion-suppression material is relatively low, mainly because the aluminum alloy explosion-suppression material is produced by the incision and stretching to form a three-dimensional cellular network structure consisting of polygonal holes ${ }^{[8-10]}$. The quality of aluminum alloy explosion-suppression material is easier to be controlled because their texture is relatively uniform, so the replacement ratio of metal explosion-suppression material is relatively lower. On the other hand, the spherical high molecular explosion-suppression material is a kind of polymer-based material. In order to suppress the dispersion of flame and explosion, the material is designed into thin sphere skeleton structure, and the spheres are closely arranged in the storage space to make the gaps among the spheres which are highly flexural, so the replacement ratio of material is relatively large. Second, the design of the diameter of the spherical material will affect the bulk density, so does the replacement ratio.

Table 1 shows that the retention ratio of polyurethane foam explosion-suppression material is as high as $3.2 \%$, mainly because the polyurethane foam explosion-suppression material is made by the way of foaming during which the rapid vaporization of water in material results in the formation of plentiful bubbles, then many cells of uneven size will form by trepan after curing process. The high specific surface area of material is acquired [11, 12] and makes the material absorb oil like a sponge. Consequently, the material produces larger retention ratio which is larger than that of other materials. However, the bone and surface of the metal explosion-suppression material and spherical high molecular explosion-suppression mate- rial are relatively smooth, and then adsorb little oil. Therefore, the retention ratio is relatively smaller. The calculated reduction ratios of effective volume of three kinds of explosion-suppression material are shown in Figure 2:

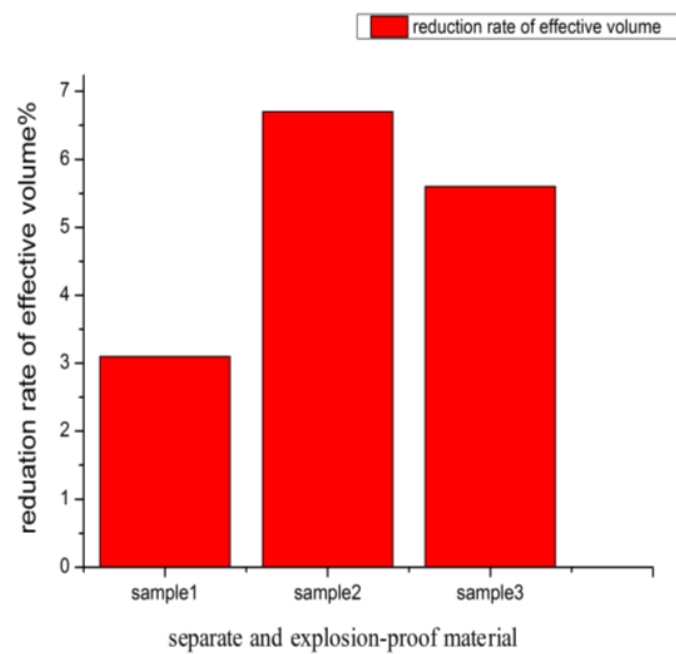

Figure 2. Reduction rate of effective volume of explosion-suppression materials

The reduction ratio of effective volume of explosion-suppression materials is considered as the basic requirement, because the effect volume of fuel tank should not be affected when filled with the explosion-suppression materials. From Figure 2, we can clearly identify that the reduction ratio of effective volume of polyurethane foam explosion-suppression material is up to $6.7 \%$, that of the aluminum alloy 


\section{MATEC Web of Conferences}

explosion-suppression material is $3.3 \%$ and that of spherical high molecular explosion-suppression material is $5.6 \%$ which is between the aluminum alloy material and the polyurethane material.

\subsection{Cook-off properties of oil tank}

The oil tank was filled with spherical high molecular explosion-suppression material and methanol. 30L diesel was added into the burning pool, and then the diesel was ignited through the ignition system. The results of the experiment are shown in Table 2.

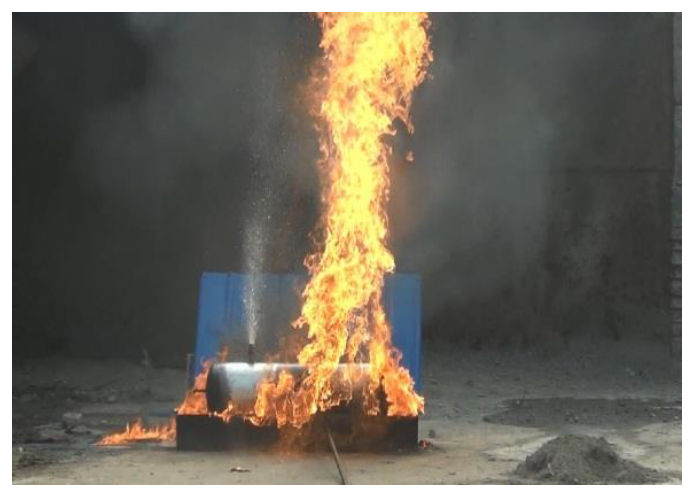

(a)

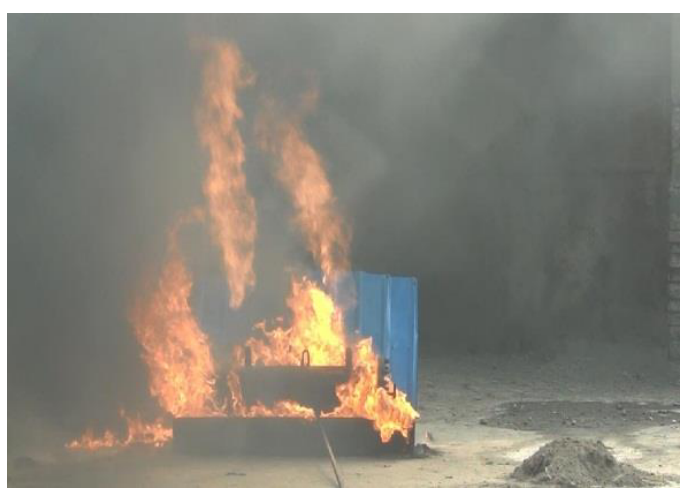

(b)

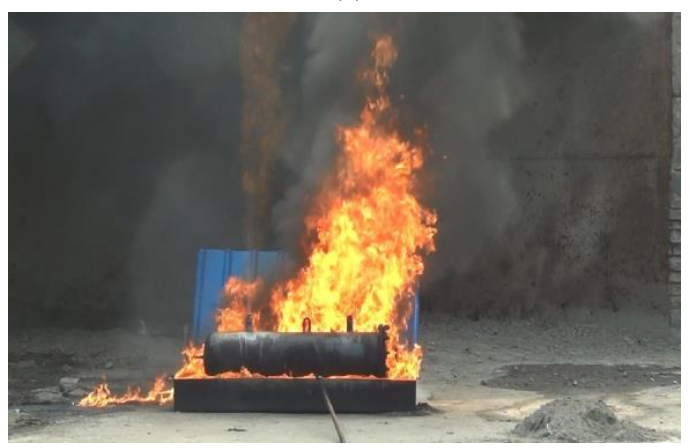

(c)

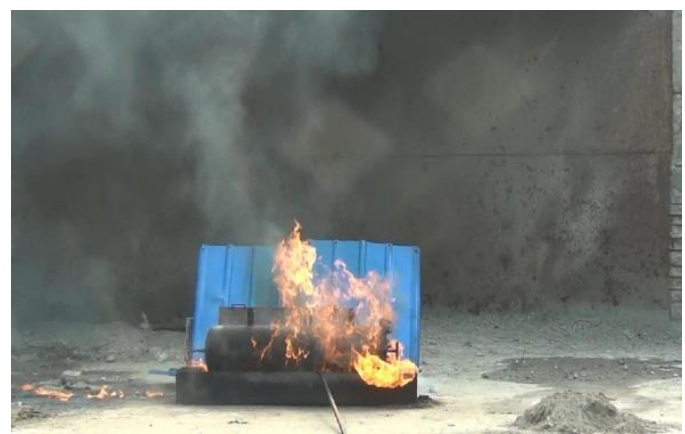

(d)

Figure 3. Character of cook-off

In the testing process, the relief valve works normally and the oil tank didn't explode. At the end of the experiments, the tank was carved open and the materials were taken out, the spherical high molecular explosion-suppression material is verified to be undamaged during the testing as shown in Figure 4:

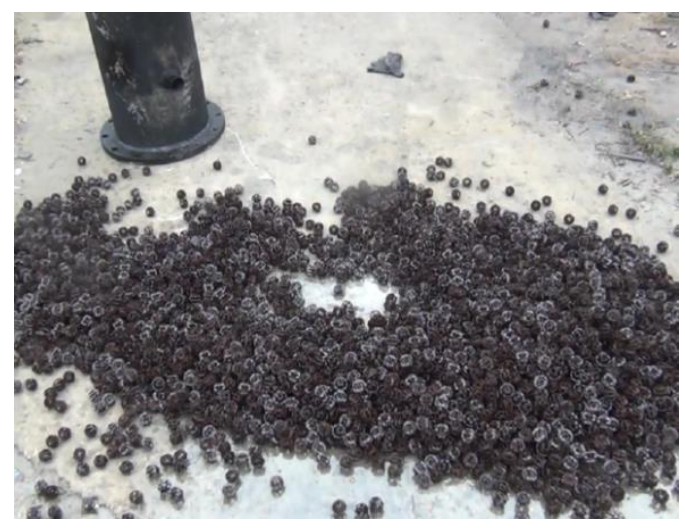

Figure 4. Spherical materials after the cook-off test

\subsection{Mechanism analysis of flame retardant and ex- plosion-suppression of explosion-suppression material}

First, according to the chain reaction theory, the combustion or explosion is not a kind of reaction occurring directly among molecules, but a kind of chain reaction proceeding among the free radicals. As the number of free radical increases, the combustion rate also increases. Flame spreads in a vessel or a pipeline filled with combustible gas mixture. When the diameter of pipeline decreases, the probability of free radicals knocking on the vessel or pipeline wall will increase. If the diameter is small enough to a critical quenching value, the flame cannot spread ${ }^{[13,14]}$. The spherical high molecular explosion-suppression material is made by selecting inert organic polymer materials as substrate and adding appropriate flame retardants, carbon fiber, thermal conductive additives and con- 
ductive additives. Furthermore, the spherical high molecular explosion-suppression material is designed as a reasonable and unique structure according to the critical quenching value of the flame. When the material is filled in a fuel tank or pipeline, the pore structure of materials will form numerous small flame channels in the fuel tank or pipeline. The inside or outside surface of flame channels will have frequent collisions with free radicals caused by combustion to weaken the energy and suppress the flame and explosion.

Second, violent combustion is generally an auto-acceleration chemical reaction in which the energy produced. It will gradually accumulate until to a degree that cannot be suppressed, i.e. explosion occurs. The specific surface area of the spherical high molecular explosion-suppression material is relatively large, namely, the radiating area is large, contributing to a cooling effect on the reaction. Meanwhile, the thermal conduction of material can transfer part of the heat to the device wall outside of the ignition zone, which can play a role in preventing flame spreading or explosion. In addition, the skeleton structure in the spherical high molecular explosion-suppression material will produce mechanical obstruction on the flame propagation. Then the pressure wave propagation can be weakened, decreasing the mixing among fuel and gas molecules, and then the risk of flame propagation and explosion development will be reduced accordingly. Through the cook-off test of the fuel tank, it can be concluded that the spherical high molecular explosion-suppression material has a good flame retardant and explosion-suppression property.

\section{CONCLUSIONS}

The reduction ratio of effective volume and cook-off properties of three kinds of explosion-suppression material which are respectively the aluminum alloy explosion-suppression material, the polyurethane foam explosion-suppression material and the spherical high molecular explosion-suppression material was studied in this paper and the following conclusions can be obtained:

1) The replacement ratio of aluminum alloy explosion-suppression material, the polyurethane foam explosion-suppression material and the spherical high molecular explosion-suppression material were respectively $2.2 \%, 2.5 \%$ and $5.2 \%$.

2) The retention ratio of polyurethane foam explosion-suppression material was relatively as high as $4.2 \%$, the retention ratio of aluminum alloy explosion-suppression material and spherical high molecular explosion-suppression material were respectively $1.1 \%$ and $0.4 \%$. The reduction ratio of effective volume of the aluminum alloy explosion-suppression material, the polyurethane foam explosion-suppression material and the spherical high molecular explosion-suppression material is respectively $3.3 \%, 6.7 \%$ and $5.6 \%$, among which the polyurethane foam explo- sion-suppression materials affect the effect volume of the fuel tank most obviously if filled with explosion-suppression materials.

3) During the cook-off testing, the methanol in the tank doesn't explode when the tank is filled with the spherical high molecular explosion-suppression material. After the cook-off testing, most of the spherical high molecular explosion-suppression materials inside the tank keep undamaged.

\section{REFERENCES}

[1] Lu Changbo, An Gaojun, \& Wang Haozhe, et al. 2014 Study on influence of explosion suppression materials on properties of oil in storage process. Journal of Safety Science and Technology, 10(10):124-130.

[2] Ke Xuan. 2005. The technology of HAN explosion suppression and safety of fuel storage. Regulations of Hazardous Chemicals, (9):80-81.

[3] Mo Suping. 2009 Explosion-suppression material. CN 101608276A, 09-23.

[4] Xiao Renliang. 2009. Explosion suppression of ball used for inflammable and explosion goods. CN: 2584220Y, 09-23.

[5] MIL-PRF-87260B (USFA), Performance Specification Foam Material, Explosion Suppression, Inherently Electrostatically Conductive for Aircraft Fuel Tank, 2006.

[6] Sechmits GT.ODTX test prom[R].MHSMP-80-40, 1980.

[7] Kent R, \& Rat M. 1982. Explosion thermique (cook-off) des propergols solides. Propellant, Explosive \& Pyrotechnical, 7:129-135.

[8] Tian Yuan, Gu Weifang, \& Tian Hong. 2007. The Performance of New Reticulate Aluminum Alloy Fire and Explosion Suppression Material and Its Application. Industrial Safety and Environmental Protection, 33(3):38-40

[9] Lu Xujie, \& Ding Bangqin. 2006. Research on aluminum-magnesium alloy explosion suppression material and its performance. Journal of Shenyang Institute of Engineering, 2(2):176-178.

[10]Birk A M. 2008. Review of expanded aluminum products for explosion suppression in containers holding flammable liquids and gas. Journal of Loss Prevention in the Process Industries, 21(5):493-505.

[11]SAE. AIR4170. Reticulated polyurethane foam explosion suppression material for fuel system and dry bays. SAE. 1998.

[12] Technical products function sheet Safety foam for explosion suppression and baffling, flexible bladders, wetwings, auxiliayr tanks, vehicle and shipboard fuel tanks, ground refuelers. Fomax International inc. 2000.

[13] Wang Dong, Xie Lifeng, \& Han Zhiwei et al. 2013. Study on Antioxidant Ability and Explosion Suppression Performance of Nonmetallic Spherical Explosion Suppression Materials. China Safety Science Journal, 23(12):66-70

[14]Ibrahim S S, \& Masri A R. 2001. The effects of obstructions on over pressure resulting from premixed flame deflagration. Journal of Loss Prevention in the Process Industries, 14(3):213-221. 